TRANSACTIONS OF THE

AMERICAN MATHEMATICAL SOCIETY

Volume 363, Number 2, February 2011, Pages 1041-1060

S 0002-9947(2010)05191-8

Article electronically published on September 14, 2010

\title{
COMBINING HOOK LENGTH FORMULAS AND BG-RANKS FOR PARTITIONS VIA THE LITTLEWOOD DECOMPOSITION
}

\author{
GUO-NIU HAN AND KATHY Q. JI
}

\begin{abstract}
Recently, the first author has studied hook length formulas for partitions in a systematic manner. In the present paper we show that most of those hook length formulas can be generalized and include more variables via the Littlewood decomposition, which maps each partition to its $t$-core and $t$-quotient. In the case $t=2$ we obtain new formulas by combining the hook lengths and BG-ranks introduced by Berkovich and Garvan. As applications, we list several multivariable generalizations of classical and new hook length formulas, including the Nekrasov-Okounkov, the Han-Carde-LoubertPotechin-Sanborn, the Bessenrodt-Bacher-Manivel, the Okada-Panova and the Stanley-Panova formulas.
\end{abstract}

\section{SUMMARY}

§1. Introduction. Main Theorems. Selected hook formulas.

$\S 2$. Combinatorial properties of the Littlewood decomposition.

$\S 3$. Generating function for partitions.

$\S 4$. Two classical hook length formulas.

$\S 5$. The Han-Carde-Loubert-Potechin-Sanborn formula.

$\S 6$. The Nekrasov-Okounkov formula.

§7. The Bessenrodt-Bacher-Manivel formula.

$\S 8$. The Okada-Panova formula.

$\S 9$. The Stanley-Panova formula.

\section{INTRODUCTION}

The hook lengths of partitions are widely studied in Partition Theory, Algebraic Combinatorics and Group Representation Theory. Recently, the first author has studied hook length formulas for partitions in a systematic manner. See 13 for the motivation of this new study of hook length formulas. In the present paper the term "hook length formula" means a formula involving the hook length of partitions in the following form:

$$
\sum_{\lambda \in \mathcal{P}} q^{|\lambda|} \prod_{h \in \mathcal{H}(\lambda)} \rho_{1}(h) \sum_{h \in \mathcal{H}(\lambda)} \rho_{2}(h)=f(q),
$$

Received by the editors March 10, 2009 and, in revised form, August 24, 2009.

2010 Mathematics Subject Classification. Primary 05A15, 05A17, 05A19, 11P81, 11P83.

Key words and phrases. Hook length formulas, BG-ranks, integer partitions, Littlewood decomposition.

The second author was partially supported by the PCSIRT Project of the Ministry of Education and the National Science Foundation of China under Grant No. 10901087.

(C)2010 American Mathematical Society 
where $\rho_{1}, \rho_{2}: \mathbb{N}^{*} \rightarrow K$ are two maps of the set of positive integers to some field $K$ and $f(q) \in K[[q]]$ is a formal power series in $q$ with coefficients in $K$ such that $f(0)=1$. In the above formula, $\mathcal{P}$ is the set of all integer partitions $\lambda$ with $|\lambda|$ denoting the integer partitioned by $\lambda$ and $\mathcal{H}(\lambda)$ the classical multiset of hook lengths associated with $\lambda$ [13. See [2, p. 1], [16, p. 1], 22, p. 287] for the basic notions on partitions. Let us list several hook length formulas having the above hook length form:

$$
\begin{aligned}
& \sum_{\lambda \in \mathcal{P}} q^{|\lambda|} \prod_{h \in \mathcal{H}(\lambda)} \frac{1}{h^{2}}=\exp (q), \\
& \sum_{\lambda \in \mathcal{P}} q^{|\lambda|} \prod_{h \in \mathcal{H}(\lambda)} \frac{1}{h}=\exp \left(q+\frac{q^{2}}{2}\right), \\
& \sum_{\lambda \in \mathcal{P}} q^{|\lambda|} \prod_{h \in \mathcal{H}(\lambda)} 1=\prod_{k \geq 1} \frac{1}{1-q^{k}}, \\
& \sum_{\lambda \in \mathcal{P}} q^{|\lambda|} \sum_{h \in \mathcal{H}(\lambda)} h^{\beta}=\prod_{m \geq 1} \frac{1}{1-q^{m}} \times \sum_{k \geq 1} k^{\beta+1} \frac{q^{k}}{1-q^{k}}, \\
& \sum_{\lambda \in \mathcal{P}} q^{|\lambda|} \prod_{h \in \mathcal{H}(\lambda)} \frac{1}{h} \frac{1+z^{h}}{1-z^{h}}=\exp \left(\frac{1+z}{1-z} q+\frac{q^{2}}{2}\right), \\
& \sum_{\lambda \in \mathcal{P}} q^{|\lambda|} \prod_{h \in \mathcal{H}(\lambda)}\left(1-\frac{z}{h^{2}}\right)=\prod_{k \geq 1}\left(1-q^{k}\right)^{z-1}, \\
& \sum_{\lambda \in \mathcal{P}} q^{|\lambda|} \prod_{h \in \mathcal{H}(\lambda)} \frac{1}{h^{2}} \sum_{h \in \mathcal{H}(\lambda)} \prod_{i=1}^{r}\left(h^{2}-i^{2}\right)=C(r) q^{r+1} \exp (q),
\end{aligned}
$$

where

$$
\begin{gathered}
C(r)=\frac{1}{2(r+1)^{2}}\left(\begin{array}{c}
2 r \\
r
\end{array}\right)\left(\begin{array}{c}
2 r+2 \\
r+1
\end{array}\right), \\
\sum_{\lambda \in \mathcal{P}} q^{|\lambda|} \prod_{h \in \mathcal{H}(\lambda)} \frac{1}{h^{2}} \sum_{h \in \mathcal{H}(\lambda)} h^{2 k}=\exp (q) \sum_{i=0}^{k} T(k+1, i+1) C(i) q^{i+1}
\end{gathered}
$$

In (1.8), $T(k, i)$ is the central factorial number defined in (9.2) and (9.3).

Formulas (1.1) and (1.2) are two well-known hook length formulas in Group Representation Theory, which could be deduced directly from the Robinson-SchenstedKnuth correspondence [13. Formula (1.3) is the traditional generating function for partitions that goes back to Euler. Formula (1.4) could be deduced from a result due to Bessenrodt [3, 5, 10]. Formula (1.5) was conjectured by the first author [1] and then proved by Carde et al. 6. Formula (1.6) was obtained by Nekrasov and Okounkov [20] and rediscovered by the first author using the hook length expansion technique [10, 13. Formula (1.7) was conjectured by Okada [23] and proved by Panova [21. Formula (1.8) was first stated by Stanley [23, 21] and generalizes the marked hook formulas [12].

Recall that a partition $\lambda$ is a $t$-core if it has no hook equal to $t$. For example, the only 2 -cores are the "staircase" partitions $(k, k-1, \ldots, 1)$. The Littlewood decomposition is a well-known bijection which maps each partition $\lambda$ to its $t$-core 
$\mu$ and $t$ partitions $\lambda^{0}, \lambda^{1}, \ldots, \lambda^{t-1}$ such that $|\lambda|=|\mu|+t \sum_{i=0}^{t-1}\left|\lambda^{i}\right|$. From this bijection, it is immediate to obtain the well-known identity [9], [15, p. 69, p. 612], [22, p. 468] for $t$-cores:

$$
\sum_{\lambda} q^{|\lambda|}=\prod_{k \geq 1} \frac{\left(1-q^{t k}\right)^{t}}{1-q^{k}}
$$

where the sum ranges over all $t$-cores. When $t=2$ it yields Gauss's identity:

$$
\sum_{k \geq 0} q^{\left(\begin{array}{c}
k+1 \\
2
\end{array}\right)}=\prod_{k \geq 1} \frac{\left(1-q^{2 k}\right)^{2}}{1-q^{k}}=\prod_{k \geq 1} \frac{1-q^{2 k}}{1-q^{2 k-1}} .
$$

Recently, Chen, Ji and Wilf [7] found that the Littlewood decomposition for $t=2$ implies the following enumerative result due to Berkovich and Garvan [4]:

$$
\sum_{\lambda \in \mathcal{P}} q^{|\lambda|} b^{\mathrm{BG}(\lambda)}=\prod_{k \geq 1} \frac{1}{\left(1-q^{2 k}\right)^{2}} \times \sum_{j=-\infty}^{+\infty} b^{j} q^{j(2 j-1)}
$$

where $\mathrm{BG}(\lambda)$ denotes the BG-rank of the partition $\lambda$ defined as follows. First, fill each box in the Ferrers diagram of $\lambda$ with alternating \pm 1 's, chessboard style, beginning with a " +1 " in the $(1,1)$ position (see Figure 1.1). The sum of these entries is the BG-rank of $\lambda$. For example, the BG-rank of $\lambda=(6,3,3,1)$ is -1 .

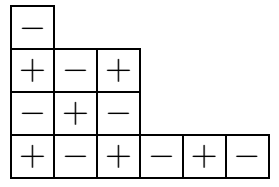

FiguRE 1.1. BG-rank

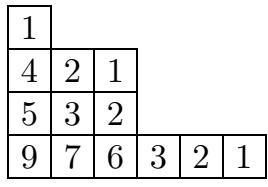

Figure 1.2. Hook lengths

In the present paper, we show that most of the hook length formulas (in particular examples (1.1)-(1.8)) can be further refined if the generating function for the $t$-core (1.9) or the BG-rank formula (1.11) are taken into account. Our main results are the following theorems, which will be proved in Section 2 by using the combinatorial properties of the Littlewood decomposition.

Theorem 1.1. If the series $f_{\alpha}(q), g_{\alpha}(q)$ and the functions $\rho_{1}(h), \rho_{2}(h)$ satisfy the relations

$$
\sum_{\lambda \in \mathcal{P}} q^{|\lambda|} \prod_{h \in \mathcal{H}(\lambda)} \rho_{1}(\alpha h)=f_{\alpha}(q)
$$

and

$$
\sum_{\lambda \in \mathcal{P}} q^{|\lambda|} \prod_{h \in \mathcal{H}(\lambda)} \rho_{1}(\alpha h) \sum_{h \in \mathcal{H}(\lambda)} \rho_{2}(\alpha h)=g_{\alpha}(q),
$$

then, for any positive integer $t$, the following identity holds:

$$
\begin{aligned}
\sum_{\lambda \in \mathcal{P}} q^{|\lambda|} x^{\# \mathcal{H}_{t}(\lambda)} \prod_{h \in \mathcal{H}_{t}(\lambda)} \rho_{1}(h) \sum_{h \in \mathcal{H}_{t}(\lambda)} \rho_{2}(h) \\
=t f_{t}\left(x q^{t}\right)^{t-1} g_{t}\left(x q^{t}\right) \prod_{k \geq 1} \frac{\left(1-q^{t k}\right)^{t}}{\left(1-q^{k}\right)}
\end{aligned}
$$

where $\mathcal{H}_{t}(\lambda)=\{h \mid h \in \mathcal{H}(\lambda), h \equiv 0(\bmod t)\}$. 
For example, the hook lengths of all boxes for the partition $\lambda=(6,3,3,1)$ have been written in each box in Figure 1.2, so that $\mathcal{H}(\lambda)=\{1,4,2,1,5,3,2$, $9,7,6,3,2,1\}$. Consequently, $\mathcal{H}_{2}(\lambda)=\{4,2,2,6,2\}$ and $\mathcal{H}_{3}(\lambda)=\{3,9,6,3\}$.

Theorem 1.2. If the series $f_{\alpha}(q), g_{\alpha}(q)$ and the functions $\rho_{1}(h), \rho_{2}(h)$ satisfy relations (1.12) and (1.13), then

$$
\begin{array}{r}
\sum_{\lambda \in \mathcal{P}} q^{|\lambda|} x^{\# \mathcal{H}_{2}(\lambda)} b^{\mathrm{BG}(\lambda)} \prod_{h \in \mathcal{H}_{2}(\lambda)} \rho_{1}(h) \sum_{h \in \mathcal{H}_{2}(\lambda)} \rho_{2}(h) \\
=2 f_{2}\left(x q^{2}\right) g_{2}\left(x q^{2}\right) \sum_{j=-\infty}^{+\infty} b^{j} q^{j(2 j-1)} .
\end{array}
$$

Let $\rho_{1}(h)=1$ in Theorems 1.1 and 1.2. Then $f_{\alpha}(q)=\prod_{k \geq 1} 1 /\left(1-q^{k}\right)$ using the generating function for partitions (1.3). Thus, we obtain the following two specializations.

Theorem 1.3 (Addition Theorem). If the series $g_{\alpha}(q)$ and the function $\rho(h)$ satisfy the relation

$$
\sum_{\lambda \in \mathcal{P}} q^{|\lambda|} \sum_{h \in \mathcal{H}(\lambda)} \rho(\alpha h)=g_{\alpha}(q)
$$

then, for any positive integer $t$,

$$
\sum_{\lambda \in \mathcal{P}} q^{|\lambda|} x^{\# \mathcal{H}_{t}(\lambda)} \sum_{h \in \mathcal{H}_{t}(\lambda)} \rho(h)=t g_{t}\left(x q^{t}\right) \prod_{k \geq 1} \frac{\left(1-q^{t k}\right)^{t}}{\left(1-\left(x q^{t}\right)^{k}\right)^{t-1}\left(1-q^{k}\right)} .
$$

Theorem 1.4 (Addition BG-Theorem). If the series $g_{\alpha}(q)$ and the function $\rho(h)$ satisfy relation (1.16), then

$$
\sum_{\lambda \in \mathcal{P}} q^{|\lambda|} x^{\# \mathcal{H}_{2}(\lambda)} b^{\mathrm{BG}(\lambda)} \sum_{h \in \mathcal{H}_{2}(\lambda)} \rho(h)=2 g_{2}\left(x q^{2}\right) \prod_{k \geq 1} \frac{1}{1-\left(x q^{2}\right)^{k}} \sum_{j=-\infty}^{+\infty} b^{j} q^{j(2 j-1)} .
$$

Let $\rho_{2}(h)=1$ in Theorems 1.1 and 1.2. Then

$$
g_{t}\left(x q^{t}\right)=x \frac{d}{d x} f_{t}\left(x q^{t}\right) .
$$

Thus, we are led to the following two results obtained by integrating both sides of (1.14) and (1.15) with respect to $x$.

Theorem 1.5 (Multiplication Theorem). If the series $f_{\alpha}(q)$ and the function $\rho(h)$ satisfy the relation

$$
\sum_{\lambda \in \mathcal{P}} q^{|\lambda|} \prod_{h \in \mathcal{H}(\lambda)} \rho(\alpha h)=f_{\alpha}(q),
$$

then, for any positive integer $t$, the following identity holds:

$$
\sum_{\lambda \in \mathcal{P}} q^{|\lambda|} x^{\# \mathcal{H}_{t}(\lambda)} \prod_{h \in \mathcal{H}_{t}(\lambda)} \rho(h)=\left(f_{t}\left(x q^{t}\right)\right)^{t} \prod_{k \geq 1} \frac{\left(1-q^{t k}\right)^{t}}{\left(1-q^{k}\right)} .
$$


Theorem 1.6 (Multiplication BG-Theorem). If the series $f_{\alpha}(q)$ and the function $\rho(h)$ satisfy relation (1.19), then

$$
\sum_{\lambda \in \mathcal{P}} q^{|\lambda|} x^{\# \mathcal{H}_{2}(\lambda)} b^{\mathrm{BG}(\lambda)} \prod_{h \in \mathcal{H}_{2}(\lambda)} \rho(h)=\left(f_{2}\left(x q^{2}\right)\right)^{2} \sum_{j=-\infty}^{+\infty} b^{j} q^{j(2 j-1)} .
$$

Take the weight function $\rho(h)=(t-h) \tilde{\rho}(h)$ with the special value $\rho(t)=0$ in Theorems 1.5 and 1.6. Then the series $f_{t}(q)$ is equal to 1 , since only the empty partition has no hook equal to one (see (1.19)). We recover the generating function (1.9) for $t$-cores from Theorem 1.5 and the following generating function for 2-cores by the BG-rank from Theorem 1.6:

$$
\sum_{\lambda: 2 \text {-core }} q^{|\lambda|} b^{\mathrm{BG}(\lambda)}=\sum_{j=-\infty}^{+\infty} b^{j} q^{j(2 j-1)} .
$$

As applications of Theorems 1.1-1.6, we derive the generalizations of formulas (1.1)-(1.8) in Sections 3-9, respectively. We should like to single out the following specializations.

Corollary 1.7 (Set $x=1$ in Theorem 3.5). We have

$$
\sum_{\lambda \in \mathcal{P}} q^{|\lambda|} y^{\#\{h \in \mathcal{H}(\lambda), h=2\}} b^{\mathrm{BG}(\lambda)} c^{\mathrm{WL}(\lambda)}=\prod_{k \geq 1} \frac{\left(1+(y-1) c q^{2 k}\right)^{2}}{\left(1-c q^{2 k}\right)^{2}} \sum_{j=-\infty}^{+\infty} b^{j} q^{j(2 j-1)},
$$

where $\mathrm{WL}(\lambda)$ is defined in (2.1).

Corollary 1.8 (Set $x=1$ and $t=2$ in Theorem 4.4). We have

$$
\sum_{\lambda \in \mathcal{P}} q^{|\lambda|} \prod_{h \in \mathcal{H}_{2}(\lambda)} \frac{1}{h}=\exp \left(q^{2}+\frac{q^{4}}{4}\right) \prod_{k \geq 1} \frac{\left(1-q^{2 k}\right)^{2}}{1-q^{k}} .
$$

Corollary 1.9 (Set $x=1$ in Theorem 5.2). We have

$$
\sum_{\lambda \in \mathcal{P}} q^{|\lambda|} b^{\mathrm{BG}(\lambda)} \prod_{h \in \mathcal{H}_{2}(\lambda)} \frac{1}{h} \frac{1+z^{h}}{1-z^{h}}=\exp \left(\frac{1+z^{2}}{1-z^{2}} q^{2}+\frac{q^{4}}{4}\right) \sum_{j=-\infty}^{+\infty} b^{j} q^{j(2 j-1)} .
$$

Corollary 1.10 (Set $x=1$ and replace $z$ by $2 z$ in Theorem 6.2). We have

$$
\sum_{\lambda \in \mathcal{P}} q^{|\lambda|} b^{\mathrm{BG}(\lambda)} \prod_{h \in \mathcal{H}_{2}(\lambda)}\left(1-\frac{2 z}{h^{2}}\right)=\prod_{k \geq 1} \frac{1}{\left(1-q^{2 k}\right)^{2-z}} \times \sum_{j=-\infty}^{+\infty} b^{j} q^{j(2 j-1)} .
$$

Corollary 1.11 (Set $\beta=-1, t=2, x=1 / q$ in Theorem 7.5). We have

$$
\sum_{\lambda \in \mathcal{P}} q^{\#\{h: \text { odd }\}} \sum_{h \in \mathcal{H}_{2}(\lambda)} \frac{1}{h}=\prod_{k \geq 1} \frac{\left(1+q^{k}\right)^{2}}{1-q^{k}} \sum_{k \geq 1} \frac{q^{k}}{1-q^{k}} .
$$

Note that the proof of Corollary 1.7 needs further combinatorial techniques developed in Theorem 2.1; it cannot be deduced from Theorem 1.6. 


\section{Combinatorial Properties of the Littlewood DeCOMposition}

In this section we prove Theorems 1.1 and 1.2 by using some combinatorial properties of a classical bijection which maps each partition to its $t$-core and $t$ quotient. This bijection probably goes back to Littlewood [17] or Nakayama [19], which will be referred to as the Littlewood decomposition. There are several ways to describe it [18, p. 12], [22, p. 468], [14, p. 75], [9]. Here we give a description in terms of binary sequences [1, [22, p. 468].

Let $\mathcal{W}$ be the set of bi-infinite binary sequences beginning with infinitely many 0 's and ending with infinitely many 1's. Each element $w$ of $\mathcal{W}$ can be represented by $\left(b_{i}\right)_{i}=\cdots b_{-3} b_{-2} b_{-1} b_{0} b_{1} b_{2} b_{3} \cdots$. However, the representation is not unique, since for any fixed integer $k$ the sequence $\left(b_{i+k}\right)_{i}$ also represents $w$. The canonical representation of $w$ is the unique sequence $\left(c_{i}\right)_{i}=\cdots c_{-3} c_{-2} c_{-1} c_{0} c_{1} c_{2} c_{3} \cdots$ such that

$$
\#\left\{i \leq-1, c_{i}=1\right\}=\#\left\{i \geq 0, c_{i}=0\right\} .
$$

It will be further denoted by $\cdots c_{-3} c_{-2} c_{-1} \cdot c_{0} c_{1} c_{2} c_{3} \cdots$ with a dot symbol inserted between the letters $c_{-1}$ and $c_{0}$.

There is a natural one-to-one correspondence between $\mathcal{P}$ and $\mathcal{W}$ (see, e.g., [1], [22, p. 468]) for more detail). Let $\lambda$ be a partition. We encode each horizontal edge of $\lambda$ by 1 and each vertical edge by 0 . Reading these $(0,1)$-encodings from top to bottom and from left to right yields a binary word $u$. By adding infinitely many 0's to the left and infinitely many 1's to the right of $u$ we get an element $w=\cdots 000 u 111 \cdots \in$ $\mathcal{W}$. Clearly, the map $\psi: \lambda \mapsto w$ is a one-to-one correspondence between $\mathcal{P}$ and $\mathcal{W}$. The canonical representation of $\psi(\lambda)$ will be denoted by $C_{\lambda}$. For example, take $\lambda=(4,3,3,1,1,1)$. Then $u=1000110010$, so that $w=\cdots 0001000110010111 \cdots$ and $C_{\lambda}=\left(c_{i}\right)_{i}=\cdots 000100011.0010111 \cdots$.

\begin{tabular}{|c|c|c|c|}
\hline 1 & & & \\
\hline 1 & \multicolumn{2}{|l|}{0} & \\
\hline 2 & \multicolumn{2}{|l|}{0} & \\
\hline 3 & \multicolumn{2}{|c|}{$\begin{array}{ll}0_{1} & 1 \\
\end{array}$} & \\
\hline 6 & 2 & 1 & 0 \\
\hline 7 & 3 & 2 & $0_{1}$ \\
\hline 9 & 5 & 4 & 1 \\
\hline
\end{tabular}

Figure 2.1. Partition and $(0,1)$-sequence

Theorem 2.1. Let $t$ be a positive integer. The Littlewood decomposition $\Omega_{t}$ maps a partition $\lambda$ to $\left(\mu ; \lambda^{0}, \lambda^{1}, \ldots, \lambda^{t-1}\right)$ such that

(P1) $\mu$ is a t-core and $\lambda^{0}, \lambda^{1}, \ldots, \lambda^{t-1}$ are partitions;

(P2) $|\lambda|=|\mu|+t\left(\left|\lambda^{0}\right|+\left|\lambda^{1}\right|+\cdots+\left|\lambda^{t-1}\right|\right)$;

(P3) $\left\{h / t \mid h \in \mathcal{H}_{t}(\lambda)\right\}=\mathcal{H}\left(\lambda^{0}\right) \cup \mathcal{H}\left(\lambda^{1}\right) \cup \cdots \cup \mathcal{H}\left(\lambda^{t-1}\right)$.

The vector $\left(\lambda^{0}, \lambda^{1}, \ldots, \lambda^{t-1}\right)$ is usually called the $t$-quotient of the partition $\lambda$.

Proof. Let us briefly describe the bijection $\Omega_{t}$ (see, e.g., [1], [22, p. 468]). Split the canonical representation $C_{\lambda}=\left(c_{i}\right)_{i}$ of the partition $\lambda$ into $t$ sections. This means that the subsequence $w^{k}=\left(c_{i t+k}\right)_{i}$ is extracted for each $k=0,1, \ldots, t-1$. The $k$-th entry $\lambda^{k}$ of the $t$-quotient of $\lambda$ is defined to be the inverse image $\psi^{-1}\left(w^{k}\right)$ 
of the subsequence $w^{k}$. With the above example $w^{0}=\cdots 00101011 \cdots$ and $w^{1}=$ $\cdots 0000100111 \cdots$, so that $\lambda^{0}=(2,1)$ and $\lambda^{1}=(1,1)$. Property (P3) holds since $\mathcal{H}\left(\lambda^{0}\right)=\{1,3,1\}, \mathcal{H}\left(\lambda^{1}\right)=\{1,2\}$ and $\mathcal{H}_{2}(\lambda)=\{2,2,6,2,4\}$ (see Figures 2.12.4). Note that the subsequence $w^{k}$ defined by $w^{k}=\left(c_{i t+k}\right)_{i}$ is not necessarily the canonical representation. For that reason we do not reproduce the dot symbol "." in the corresponding rows in the following table.

\begin{tabular}{c|ccccccccccccccccccccc}
$C_{\lambda}$ & $\cdots$ & 0 & 0 & 0 & 1 & 0 & 0 & 0 & 1 & 1 &. & 0 & 0 & 1 & 0 & 1 & 1 & 1 & 1 & 1 & $\cdots$ \\
\hline$w^{0}$ & $\cdots$ & & 0 & & 1 & & 0 & & 1 & & 0 & & 1 & & 1 & & 1 & & 1 & $\cdots$ \\
$v^{0}$ & $\cdots$ & & 0 & 0 & & 0 & & 1 & & 1 & & 1 & & 1 & & 1 & & 1 & $\cdots$ \\
\hline$w^{1}$ & $\cdots$ & 0 & & 0 & 0 & & 0 & & 1 & & 0 & 0 & & 1 & & 1 & & $\cdots$ \\
$v^{1}$ & $\cdots$ & 0 & & 0 & & 0 & & 0 & 0 & & 0 & 1 & & 1 & & 1 & & $\cdots$ \\
\hline$C_{\mu}$ & $\cdots$ & 0 & 0 & 0 & 0 & 0 & 0 & 0 & 1 & 0 & & 1 & 0 & 1 & 1 & 1 & 1 & 1 & 1 & 1 & $\cdots$
\end{tabular}

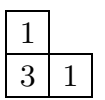

Figure 2.2. Partition $\lambda^{0}$ Figure 2.3. Partition $\lambda^{1}$

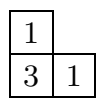

Figure 2.4. The 2-core $\mu$

For each subsequence $w^{k}$ we continually replace the subword 10 by 01 . The final resulting sequence is of the form $\cdots 000111 \cdots$ and is denoted by $v^{k}$. The $t$-core of the partition $\lambda$ is defined to be the partition $\mu$ such that the $t$ sections of the canonical representation $C_{\mu}$ are exactly $v^{0}, v^{1}, \ldots, v^{t-1}$. With the above example we have $\mu=(2,1)$. Properties (P2) and (P3) can be derived from the following basic fact: each box of $\lambda$ is in one-to-one correspondence with the ordered pair of integers $(i, j)$ such that $i<j$ and $c_{i}=1, c_{j}=0$ and the hook length of that box is equal to $j-i$.

The process above is reversible. Given $t+1$ partitions $\left(\mu ; \lambda^{0}, \lambda^{1}, \ldots, \lambda^{t-1}\right)$, where $\mu$ is a $t$-core, we first split the canonical representation $C_{\mu}=\left(d_{i}\right)_{i}$ of the partition $\mu$ into $t$ sections; namely, we form the subsequence $v^{k}=\left(d_{i t+k}\right)_{i}$ for $k=0,1, \ldots, t-1$. Clearly, every subsequence $v^{k}$ is of the form $\cdots 000111 \cdots$, since $\mu$ is a $t$-core. Let $w^{k}=\psi\left(\lambda^{k}\right)$. Note that the representation of $w^{k}$ is not unique. We choose the one which can be obtained by continually replacing the subword 01 of $v^{k}$ by 10. We then obtain the partition $\lambda$ whose canonical representation $C_{\lambda}=\left(c_{i}\right)_{i}$ consists of such sequences $w^{0}, w^{1}, \ldots, w^{t-1}$. This means that $\left(c_{i t+k}\right)_{i}=w^{k}$ for $k=0,1, \ldots, t-1$.

For the case $t=2$, the Littlewood decomposition $\Omega_{2}$ has more combinatorial properties in addition to (P1)-(P3). To describe our new properties, we need a new statistic called the weak length $\mathrm{WL}(\lambda)$ defined by means of the multiplicity notation

$$
\lambda=\left\langle 1^{m_{1}}, 2^{m_{2}}, \ldots, r^{m_{r}}\right\rangle
$$

of the partition $\lambda$. Recall that this means that exactly $m_{i}$ of the parts of $\lambda$ are equal to $i$, so that $\ell(\lambda)=\sum_{j=1}^{r} m_{j}$. Let $k$ be the smallest part such that $m_{k}$ is even. Then, the weak length $\mathrm{WL}(\lambda)$ of $\lambda$ is defined to be

$$
\mathrm{WL}(\lambda)=\sum_{j=1}^{k-1}\left(\frac{m_{j}-1}{2}\right)+\frac{m_{k}}{2}+\sum_{j=k+1}^{r} m_{j} .
$$

For example, the weak length of $\lambda=(4,3,3,1,1,1)=\left\langle 1^{3}, 2^{0}, 3^{2}, 4^{1}\right\rangle$ is 4 . 
Theorem 2.2. When $t=2$, the Littlewood decomposition $\Omega_{2}$ has the further two properties:

(P4) $\operatorname{BG}(\lambda)= \begin{cases}\frac{\ell(\mu)+1}{2} & \text { if } \mathrm{BG}(\lambda)>0, \\ -\frac{\ell(\mu)}{2} & \text { if } \quad \mathrm{BG}(\lambda) \leq 0 .\end{cases}$

(P5) $\mathrm{WL}(\lambda)=\ell\left(\lambda^{0}\right)+\ell\left(\lambda^{1}\right)$.

Proof. From the description of the Littlewood decomposition $\Omega_{2}$, it follows that the 2-core $\mu$ of the partition $\lambda$ is obtained from the canonical representation $C_{\lambda}=\left(c_{i}\right)_{i}$ by continually choosing $i$ with $c_{i}=1$ and $c_{i+2}=0$, and then replacing $c_{i}$ by 0 and $c_{i+2}$ by 1 . Such an operation is equivalent to removing a horizontal or vertical pair of adjacent cells from the Young diagram of $\lambda$. The BG-rank of the partition $\lambda$ and of its 2-core $\mu$ are then equal. On the other hand, the BG-rank of a 2-core of length $k$ is $(k+1) / 2$ if $k$ is odd, and $-k / 2$ if $k$ is even [7. This shows that Property (P4) holds.

Given a partition $\lambda=\left\langle 1^{m_{1}}, 2^{m_{2}}, \ldots, h^{m_{h}}\right\rangle$ let $u=\left(u_{i}\right)_{i \geq 0}$ be the $(0,1)$-encoding of $\lambda$. Then $u$ is the sequence of the form

$$
u=1 \underbrace{00 \cdots}_{m_{1}} 1 \underbrace{00 \cdots}_{m_{2}} 1 \underbrace{00 \cdots}_{m_{3}} 1 \underbrace{\cdots \cdots \cdots}_{\cdots} 1 \underbrace{00 \cdots 0}_{m_{h}}
$$

Clearly, the number of 0 's in $u$ is equal to the length $\ell(\lambda)$ of $\lambda$. Let $m$ be the smallest positive integer such that $u_{2 m+1} \neq 0$, so that $u_{2 j+1}=0$ for $0 \leq j \leq m-1$. From the description of the Littlewood decomposition $\Omega_{2}$ given in the proof of Theorem 2.1, it follows that the sum of the lengths of $\lambda^{0}$ and $\lambda^{1}$ is equal to $\ell(\lambda)-m$. On the other hand, let $k$ be the smallest part of $\lambda$ such that its multiplicity $m_{k}$ is even. From (2.2), it follows that $m=\left(\sum_{j=1}^{k} m_{j}+k-1\right) / 2$, where $m$ is the smallest positive integer such that $u_{2 m+1} \neq 0$. Thus, Property (P5) holds by an easy calculation.

We are ready to prove Theorems 1.1 and 1.2 by using the above properties of the Littlewood decomposition.

Proof of Theorem 1.1. By properties (P1)-(P3) of the Littlewood decomposition $\Omega_{t}$ in Theorem 2.1 we have

$$
\begin{aligned}
& \sum_{\lambda \in \mathcal{P}} q^{|\lambda|} x^{\# \mathcal{H}_{t}(\lambda)} \prod_{h \in \mathcal{H}_{t}(\lambda)} \rho_{1}(h) \sum_{h \in \mathcal{H}_{t}(\lambda)} \rho_{2}(h) \\
= & \prod_{k \geq 1} \frac{\left(1-q^{t k}\right)^{t}}{1-q^{k}} \sum_{i=0}^{t-1}\left(\sum_{\lambda \in \mathcal{P}} q^{t|\lambda|} x^{\# \mathcal{H}(\lambda)} \prod_{h \in \mathcal{H}(\lambda)} \rho_{1}(t h)\right)^{t-1} \\
& \times\left(\sum_{\lambda^{i} \in \mathcal{P}} q^{t\left|\lambda^{i}\right|} x^{\# \mathcal{H}\left(\lambda^{i}\right)} \prod_{h \in \mathcal{H}\left(\lambda^{i}\right)} \rho_{1}(t h) \sum_{h \in \mathcal{H}\left(\lambda^{i}\right)} \rho_{2}(t h)\right) \\
= & \prod_{k \geq 1} \frac{\left(1-q^{t k}\right)^{t}}{1-q^{k}} \sum_{i=0}^{t-1} f_{t}\left(x q^{t}\right)^{t-1} g_{t}\left(x q^{t}\right) \\
= & t f_{t}\left(x q^{t}\right)^{t-1} g_{t}\left(x q^{t}\right) \prod_{k \geq 1} \frac{\left(1-q^{t k}\right)^{t}}{1-q^{k}},
\end{aligned}
$$

where the third equation follows from formulas (1.12) and (1.13) by replacing $q$ by $x q^{t}$ and setting $\alpha=t$. 
Proof of Theorem 1.2. From properties (P1)-(P4) of the Littlewood decomposition $\Omega_{2}$ in Theorems 2.1 and 2.2 , we get

$$
\begin{aligned}
& \sum_{\lambda \in \mathcal{P}} q^{|\lambda|} x^{\# \mathcal{H}_{2}(\lambda)} b^{\mathrm{BG}(\lambda)} \prod_{h \in \mathcal{H}_{2}(\lambda)} \rho_{1}(h) \sum_{h \in \mathcal{H}_{2}(\lambda)} \rho_{2}(h) \\
& =\sum_{j=-\infty}^{+\infty} b^{j} q^{j(2 j-1)} \times 2\left(\sum_{\lambda \in \mathcal{P}} q^{2|\lambda|} x^{\# \mathcal{H}(\lambda)} \prod_{h \in \mathcal{H}(\lambda)} \rho_{1}(2 h)\right) \\
& \times\left(\sum_{\lambda \in \mathcal{P}} q^{2|\lambda|} x^{\# \mathcal{H}(\lambda)} \prod_{h \in \mathcal{H}(\lambda)} \rho_{1}(2 h) \sum_{h \in \mathcal{H}(\lambda)} \rho_{2}(2 h)\right) \\
& =2 f_{2}\left(x q^{2}\right) g_{2}\left(x q^{2}\right) \sum_{j=-\infty}^{+\infty} b^{j} q^{j(2 j-1)},
\end{aligned}
$$

where the last equation follows from formulas (1.12) and (1.13) by replacing $q$ by $x q^{2}$ and setting $\alpha=2$.

\section{Generating Function for partitions}

In this section we give several generalizations of generating function for partitions (1.3). First, we obtain the following two generalizations by applying Theorems 1.5 and 1.6 with the weight function $\rho(h)=1$.

Theorem 3.1 ([12, Corollary 5.1]). We have

$$
\sum_{\lambda \in \mathcal{P}} q^{|\lambda|} x^{\# \mathcal{H}_{t}(\lambda)}=\prod_{k \geq 1} \frac{\left(1-q^{t k}\right)^{t}}{\left(1-\left(x q^{t}\right)^{k}\right)^{t}\left(1-q^{k}\right)} .
$$

Theorem 3.2. We have

$$
\sum_{\lambda \in \mathcal{P}} q^{|\lambda|} x^{\# \mathcal{H}_{2}(\lambda)} b^{\mathrm{BG}(\lambda)}=\prod_{k \geq 1} \frac{1}{\left(1-\left(x q^{2}\right)^{k}\right)^{2}} \sum_{j=-\infty}^{+\infty} b^{j} q^{j(2 j-1)} .
$$

In fact, we can obtain more generalizations by means of the combinatorial properties of the Littlewood decomposition. Before doing this, we need the following lemma.

Lemma 3.3. We have

$$
\sum_{\lambda \in \mathcal{P}} q^{|\lambda|} y^{\#\{h \in \mathcal{H}(\lambda), h=1\}} c^{\ell(\lambda)}=\prod_{k \geq 1} \frac{1+(y-1) c q^{k}}{1-c q^{k}} .
$$

Proof.

$$
\begin{aligned}
\sum_{\lambda \in \mathcal{P}} q^{|\lambda|} y^{\#\{h \in \mathcal{H}(\lambda), h=1\}} c^{\ell(\lambda)} & =\prod_{k \geq 1}\left(1+y c q^{k}+y c^{2} q^{2 k}+y c^{3} q^{3 k}+\cdots\right) \\
& =\prod_{k \geq 1}\left(1+\frac{y c q^{k}}{1-c q^{k}}\right)=\prod_{k \geq 1} \frac{1+(y-1) c q^{k}}{1-c q^{k}} .
\end{aligned}
$$

The following theorem unifies Theorem 3.1 and another result of the first author 12, Theorem 1.4]. 
Theorem 3.4. We have

$$
\sum_{\lambda \in \mathcal{P}} q^{|\lambda|} x^{\# \mathcal{H}_{t}(\lambda)} y^{\#\{h \in \mathcal{H}(\lambda), h=t\}}=\prod_{k \geq 1} \frac{\left(1-q^{t k}\right)^{t}\left(1+(y-1)\left(x q^{t}\right)^{k}\right)^{t}}{\left(1-q^{k}\right)\left(1-\left(x q^{t}\right)^{k}\right)^{t}} .
$$

Proof. From properties (P1)-(P3) of the bijection $\Omega_{t}$ in Theorem 2.1 we get

$$
\begin{aligned}
& \sum_{\lambda \in \mathcal{P}} q^{|\lambda|} x^{\# \mathcal{H}_{t}(\lambda)} y^{\#\{h \in \mathcal{H}(\lambda), h=t\}} \\
= & \prod_{k \geq 1} \frac{\left(1-q^{t k}\right)^{t}}{1-q^{k}} \times\left(\sum_{\lambda \in \mathcal{P}} q^{t|\lambda|} x^{\# \mathcal{H}(\lambda)} y^{\#\{h \in \mathcal{H}(\lambda), h=1\}}\right)^{t} \\
= & \prod_{k \geq 1} \frac{\left(1-q^{t k}\right)^{t}}{1-q^{k}} \times\left(\sum_{\lambda \in \mathcal{P}}\left(x q^{t}\right)^{|\lambda|} y^{\#\{h \in \mathcal{H}(\lambda), h=1\}}\right)^{t} \\
= & \prod_{k \geq 1} \frac{\left(1-q^{t k}\right)^{t}\left(1+(y-1)\left(x q^{t}\right)^{k}\right)^{t}}{\left(1-q^{k}\right)\left(1-\left(x q^{t}\right)^{k}\right)^{t}},
\end{aligned}
$$

where the last identity follows from Lemma 3.3 by replacing $q$ by $x q^{2}$ and setting $c=1$.

The following theorem gives a generalization of Theorem 3.2.

Theorem 3.5. We have

$$
\begin{aligned}
& \sum_{\lambda \in \mathcal{P}} q^{|\lambda|} x^{\# \mathcal{H}_{2}(\lambda)} y^{\#\{h \in \mathcal{H}(\lambda), h=2\}} b^{\mathrm{BG}(\lambda)} c^{\mathrm{WL}(\lambda)} \\
&=\prod_{k \geq 1} \frac{\left(1+(y-1) c\left(x q^{2}\right)^{k}\right)^{2}}{\left(1-c\left(x q^{2}\right)^{k}\right)^{2}} \sum_{j=-\infty}^{+\infty} b^{j} q^{j(2 j-1)} .
\end{aligned}
$$

Proof. From properties (P1)-(P5) of the bijection $\Omega_{2}$ in Theorems 2.1 and 2.2 we get

$$
\begin{aligned}
& \sum_{\lambda \in \mathcal{P}} q^{|\lambda|} x^{\# \mathcal{H}_{2}(\lambda)} y^{\#\{h \in \mathcal{H}(\lambda), h=2\}} b^{\mathrm{BG}(\lambda)} c^{\mathrm{WL}(\lambda)} \\
= & \left(\sum_{\lambda \in \mathcal{P}} q^{2|\lambda|} x^{\# \mathcal{H}(\lambda)} y^{\#\{h \in \mathcal{H}(\lambda), h=1\}} c^{\ell(\lambda)}\right)^{2} \times \sum_{j=-\infty}^{+\infty} b^{j} q^{j(2 j-1)} \\
= & \left(\sum_{\lambda \in \mathcal{P}}\left(x q^{2}\right)^{|\lambda|} y^{\#\{h \in \mathcal{H}(\lambda), h=1\}} c^{\ell(\lambda)}\right)^{2} \times \sum_{j=-\infty}^{+\infty} b^{j} q^{j(2 j-1)} \\
= & \prod_{k \geq 1} \frac{\left(1+(y-1) c\left(x q^{2}\right)^{k}\right)^{2}}{\left(1-c\left(x q^{2}\right)^{k}\right)^{2}} \sum_{j=-\infty}^{+\infty} b^{j} q^{j(2 j-1)}
\end{aligned}
$$

where the last identity follows from Lemma 3.3 by replacing $q$ by $x q^{2}$.

Setting $x=y=b=1$ in Theorem 3.5 and using Gauss' identity (1.10), we obtain the following corollary. 
Corollary 3.6. We have

$$
\sum_{\lambda \in \mathcal{P}} q^{|\lambda|} c^{\mathrm{WL}(\lambda)}=\prod_{k \geq 1} \frac{\left(1-q^{2 k}\right)}{\left(1-q^{2 k-1}\right)\left(1-c q^{2 k}\right)^{2}} .
$$

By definition (2.1) of the weak length $\mathrm{WL}(\lambda)$ we also have

$$
\begin{aligned}
\sum_{\lambda \in \mathcal{P}} q^{|\lambda|} c^{\mathrm{WL}(\lambda)=} \sum_{k \geq 1} \prod_{j=1}^{k-1}\left(q^{j}+c q^{3 j}+c^{2} q^{5 j}+\cdots\right) \\
\times\left(1+c q^{2 k}+c^{2} q^{4 k}+\cdots\right) \\
\times \prod_{j=k+1}^{+\infty}\left(1+c q^{j}+c^{2} q^{2 j}+\cdots\right) \\
=\sum_{k \geq 1} q^{\left(\begin{array}{l}
k \\
2
\end{array}\right)} \prod_{j=1}^{k} \frac{1}{1-c q^{2 j}} \prod_{j=k+1}^{+\infty} \frac{1}{1-c q^{j}} \\
=\prod_{j=1}^{+\infty} \frac{1}{1-c q^{j}} \sum_{k \geq 1} q^{\left(\begin{array}{c}
k \\
2
\end{array}\right)} \prod_{j=1}^{k} \frac{1-c q^{j}}{1-c q^{2 j}} .
\end{aligned}
$$

Combining (3.1) and (3.2) we get the following identity, which is a special case of the $q$-Gauss second identity [8, p. 237, Eq.(II.11)] for $a=\sqrt{c} q, b=\sqrt{q}$ :

$$
\sum_{k \geq 0} q^{\left(\begin{array}{c}
k+1 \\
2
\end{array}\right)} \prod_{j=1}^{k+1} \frac{1-c q^{j}}{1-c q^{2 j}}=\prod_{k \geq 1} \frac{\left(1-c q^{2 k-1}\right)\left(1-q^{2 k}\right)}{\left(1-q^{2 k-1}\right)\left(1-c q^{2 k}\right)} .
$$

\section{Two Classical hoOK LENGTh Formulas}

The two multiplication theorems are also applicable to the two classical hook length formulas (1.1) and (1.2) [13, themselves obtainable from the RobinsonSchensted-Knuth correspondence (see, for example, [15, pp. 49-59], 222, p. 324]). When applying Theorems 1.5 and 1.6 with the weight function $\rho(h)=1 / h^{2}$, we get the following generalizations of (1.1).

Theorem 4.1 ([12, Corollary 5.4]). We have

$$
\sum_{\lambda \in \mathcal{P}} q^{|\lambda|} x^{\# \mathcal{H}_{t}(\lambda)} \prod_{h \in \mathcal{H}_{t}(\lambda)} \frac{1}{h^{2}}=\exp \left(\frac{x q^{t}}{t}\right) \prod_{k \geq 1} \frac{\left(1-q^{t k}\right)^{t}}{1-q^{k}} .
$$

Theorem 4.2. We have

$$
\sum_{\lambda \in \mathcal{P}} q^{|\lambda|} x^{\# \mathcal{H}_{2}(\lambda)} b^{\mathrm{BG}(\lambda)} \prod_{h \in \mathcal{H}_{2}(\lambda)} \frac{1}{h^{2}}=\exp \left(\frac{x q^{2}}{2}\right) \sum_{j=-\infty}^{+\infty} b^{j} q^{j(2 j-1)} .
$$

The following result is immediate from Theorem 4.2 by setting $x=1$ and comparing the coefficients of $b^{0}$ on both sides.

Corollary 4.3. We have

$$
\sum_{\lambda \in \mathcal{P}, \operatorname{BG}(\lambda)=0} q^{|\lambda|} \prod_{h \in \mathcal{H}_{2}(\lambda)} \frac{1}{h^{2}}=\exp \left(\frac{q^{2}}{2}\right) .
$$


Corollary 4.3 could also be derived from formula (1.5) by setting $z=-1$. Note that, for any partition $\lambda$, the number of odd hooks in $\lambda$ is greater than or equal to the number of even hooks in $\lambda$. Moreover, when the number of odd hooks and the number of even hooks in $\lambda$ are equal, the BG-rank of $\lambda$ is equal to 0 .

The following theorems are generalizations of (1.2). They are immediate consequences of Theorems 1.5 and 1.6 with the weight function $\rho(h)=1 / h$ :

Theorem 4.4. We have

$$
\sum_{\lambda \in \mathcal{P}} q^{|\lambda|} x^{\# \mathcal{H}_{t}(\lambda)} \prod_{h \in \mathcal{H}_{t}(\lambda)} \frac{1}{h}=\exp \left(x q^{t}+\frac{x^{2} q^{2 t}}{2 t}\right) \prod_{k \geq 1} \frac{\left(1-q^{t k}\right)^{t}}{1-q^{k}} .
$$

Theorem 4.5. We have

$$
\sum_{\lambda \in \mathcal{P}} q^{|\lambda|} x^{\# \mathcal{H}_{2}(\lambda)} b^{\mathrm{BG}(\lambda)} \prod_{h \in \mathcal{H}_{2}(\lambda)} \frac{1}{h}=\exp \left(x q^{2}+\frac{x^{2} q^{4}}{4}\right) \sum_{j=-\infty}^{+\infty} b^{j} q^{j(2 j-1)} .
$$

By comparing the coefficients of $x^{n} q^{t n}$ on both sides of (4.1) we have the following result.

Corollary 4.6. We have

$$
\sum_{\lambda \vdash t n, \# \mathcal{H}_{t}(\lambda)=n} \prod_{h \in \mathcal{H}_{t}(\lambda)} \frac{1}{h}=\sum_{j=0}^{\left\lfloor\frac{n}{2}\right\rfloor} \frac{1}{j !(n-2 j) !(2 t)^{j}} .
$$

Setting $x=1$ and comparing the coefficients of $b^{0}$ on both sides of (4.2) yields the following result.

Corollary 4.7. We have

$$
\sum_{\lambda \in \mathcal{P}, \mathrm{BG}(\lambda)=0} q^{|\lambda|} \prod_{h \in \mathcal{H}_{2}(\lambda)} \frac{1}{h}=\exp \left(q^{2}+\frac{q^{4}}{4}\right)
$$

\section{The Han-Carde-Loubert-Potechin-Sanborn formula}

In [11, the first author found the hook length formula (1.5), related to partitions and permutations. Formula (1.5) has recently been proved by Carde et al. 6]. Formula (1.5) is an interpolation between the two classical hook length formulas. It reduces to (1.2) when $z=0$. When $q$ is replaced by $\frac{1-z}{1+z} q$ and $z$ is equal to 1 , formula (1.5) yields (1.1).

When $\rho(h)=\left(1+z^{h}\right) /\left(h\left(1-z^{h}\right)\right)$, the series $f_{\alpha}(q)$ defined in (1.19) has the following explicit form:

$$
f_{\alpha}(q)=\sum_{\lambda \in \mathcal{P}} q^{|\lambda|} \prod_{h \in \mathcal{H}(\lambda)} \frac{1}{\alpha h} \frac{1+z^{\alpha h}}{1-z^{\alpha h}}=\exp \left(\frac{1+z^{\alpha}}{1-z^{\alpha}} \frac{q}{\alpha}+\frac{q^{2}}{2 \alpha^{2}}\right) .
$$

Hence, Theorems 1.5 and 1.6 imply the following generalizations of (1.5).

Theorem 5.1. Let $t$ be a positive integer. Then

$$
\sum_{\lambda \in \mathcal{P}} q^{|\lambda|} x^{\# \mathcal{H}_{t}(\lambda)} \prod_{h \in \mathcal{H}_{t}(\lambda)} \frac{1}{h} \frac{1+z^{h}}{1-z^{h}}=\exp \left(\frac{1+z^{t}}{1-z^{t}} x q^{t}+\frac{x^{2} q^{2 t}}{2 t}\right) \prod_{k \geq 1} \frac{\left(1-q^{t k}\right)^{t}}{\left(1-q^{k}\right)}
$$


Theorem 5.2. We have

$$
\begin{aligned}
\sum_{\lambda \in \mathcal{P}} q^{|\lambda|} x^{\# \mathcal{H}_{2}(\lambda)} b^{\mathrm{BG}(\lambda)} \prod_{h \in \mathcal{H}_{2}(\lambda)} \frac{1}{h} \frac{1+z^{h}}{1-z^{h}} \\
=\exp \left(\frac{1+z^{2}}{1-z^{2}} x q^{2}+\frac{x^{2} q^{4}}{4}\right) \sum_{j=-\infty}^{+\infty} b^{j} q^{j(2 j-1)} .
\end{aligned}
$$

When $z=0$, Theorems 5.1 and 5.2 reduce to Theorems 4.4 and 4.5, respectively. When $x$ is replaced by $\frac{1-z}{1+z} x$ and $z=1$, Theorems 5.1 and 5.2 give back Theorems 4.1 and 4.2 , respectively.

\section{The Nekrasov-Okounkov formula}

Formula (1.6) can be seen as an explicit expansion formula for the powers of the Euler Product in terms of partition hook lengths. It was first discovered by Nekrasov and Okounkov in their study of the Seiberg-Witten theory and random partitions [20] and rediscovered by the first author [10] by means of an appropriate hook length expansion technique [13. An elementary proof of the NekrasovOkounkov formula is given in [12].

Theorems 1.5 and 1.6 with the weight function $\rho(h)=1-z / h^{2}$ yield the following generalizations of (1.6).

Theorem 6.1 ([12, Theorem 1.2]). We have

$$
\sum_{\lambda \in \mathcal{P}} q^{|\lambda|} x^{\# \mathcal{H}_{t}(\lambda)} \prod_{h \in \mathcal{H}_{t}(\lambda)}\left(1-\frac{z}{h^{2}}\right)=\prod_{k \geq 1} \frac{\left(1-q^{t k}\right)^{t}}{\left(1-\left(x q^{t}\right)^{k}\right)^{t-z / t}\left(1-q^{k}\right)} .
$$

Theorem 6.2. We have

$$
\begin{aligned}
\sum_{\lambda \in \mathcal{P}} q^{|\lambda|} x^{\# \mathcal{H}_{2}(\lambda)} b^{\mathrm{BG}(\lambda)} \prod_{h \in \mathcal{H}_{2}(\lambda)}\left(1-\frac{z}{h^{2}}\right) \\
=\prod_{k \geq 1} \frac{1}{\left(1-\left(x q^{2}\right)^{k}\right)^{2-z / 2}} \times \sum_{j=-\infty}^{+\infty} b^{j} q^{j(2 j-1)} .
\end{aligned}
$$

Corollary 6.3. We have

$$
\sum_{\lambda \vdash 2 n+j(2 j-1), \operatorname{BG}(\lambda)=j} \prod_{h \in \mathcal{H}_{2}(\lambda)} \frac{1}{h^{2}} \sum_{h \in \mathcal{H}_{2}(\lambda)} h^{2}=\frac{3 n+1}{2^{n}(n-1) !} .
$$

Proof. Clearly, the left-hand side of (6.3) is the coefficient of

$$
q^{2 n+j(2 j-1)} b^{j} x^{n}(-z)^{n-1}
$$

on the left-hand side of (6.2). Using the following identity,

$$
\prod_{m \geq 1} \frac{1}{1-q^{m}}=\exp \left(\sum_{k \geq 1} \frac{q^{k}}{k\left(1-q^{k}\right)}\right)
$$

the right-hand side of (6.2) can be written:

$$
R=\prod_{k \geq 1} \frac{1}{\left(1-\left(x q^{2}\right)^{k}\right)^{2}} \exp \left(\frac{-z}{2} \sum_{k \geq 1} \frac{\left(x q^{2}\right)^{k}}{k\left(1-\left(x q^{2}\right)^{k}\right)}\right) \sum_{j=-\infty}^{+\infty} b^{j} q^{j(2 j-1)} .
$$


Thus,

$$
\begin{aligned}
& {\left[q^{2 n+j(2 j-1)} b^{j} x^{n}(-z)^{n-1}\right] R } \\
= & {\left[q^{2 n} x^{n}(-z)^{n-1}\right] \prod_{k \geq 1} \frac{1}{\left(1-\left(x q^{2}\right)^{k}\right)^{2}} \exp \left(\frac{-z}{2} \sum_{k \geq 1} \frac{\left(x q^{2}\right)^{k}}{k\left(1-\left(x q^{2}\right)^{k}\right)}\right) } \\
= & {\left[q^{2 n} x^{n}\right] \frac{1}{2^{n-1}(n-1) !} \prod_{k \geq 1} \frac{1}{\left(1-\left(x q^{2}\right)^{k}\right)^{2}}\left(\sum_{k \geq 1} \frac{\left(x q^{2}\right)^{k}}{k\left(1-\left(x q^{2}\right)^{k}\right)}\right)^{n-1} } \\
= & {\left[q^{2} x\right] \frac{1}{2^{n-1}(n-1) !} \frac{1}{\left(1-\left(x q^{2}\right)\right)^{2}}\left(\frac{1}{\left(1-\left(x q^{2}\right)\right.}+\frac{x q^{2}}{2\left(1-\left(x q^{2}\right)^{2}\right)}\right)^{n-1} } \\
= & \frac{1}{2^{n-1}(n-1) !}\left(2+n-1+\frac{n-1}{2}\right) \\
= & \frac{3 n+1}{2^{n}(n-1) !} .
\end{aligned}
$$

Corollary 6.3 could also be derived from Theorem 9.2 by setting $x=1, k=1$ and comparing the coefficients of $b^{j} q^{2 n+j(2 j-1)}$ on both sides.

Setting $x=1$ and comparing the coefficients of $(-z) b^{j}$ on both sides of (6.2) leads to the following corollary.

Corollary 6.4. We have

$$
\sum_{\lambda \in \mathcal{P}, \mathrm{BG}(\lambda)=j} q^{|\lambda|} \sum_{h \in \mathcal{H}_{2}(\lambda)} \frac{1}{h^{2}}=\frac{q^{j(2 j-1)}}{2 \prod_{k \geq 1}\left(1-q^{2 k}\right)^{2}} \sum_{k \geq 1} \frac{q^{2 k}}{k\left(1-q^{2 k}\right)} .
$$

Corollary 6.4 could also be deduced from Theorem 7.6 by setting $\beta=-2, x=1$, and comparing the coefficients of $b^{j}$ on both sides.

\section{The Bessenrodt-Bacher-Manivel formula}

Formula (1.4) deals with power sums of hook lengths. Its proof is based on an elegant result about the multi-set of hook lengths and the multi-set of parts of all partitions of $n$ due to Bessenrodt, Bacher and Manivel [3, 5. See also [10] for some historical remarks. Each hook length $h$ can be split into $h=a+l+1$, where $a$ is the arm length and $l$ the leg length (see [22, p. 457]). The ordered pair $(a, l)$ is called a hook type.

Theorem 7.1 (Bessenrodt-Bacher-Manivel). Let $n \geq k \geq 1$ be two integers. Then, for every positive $j<k$, the total number of occurrences of the part $k$ among all partitions of $n$ is equal to the number of boxes, whose hook type is $(j, k-j-1)$.

Theorem 7.1 implies in particular that the total number of hooks of given hook type $(j, k-j-1)$ occurring in all partitions of $n$ depends only on the length $k$ and not on the particular hook type itself. Since there are exactly $k$ distinct hook types for hooks of length $k$, the total number of hooks of length $k$ in all partitions of $n$ is $k$ times the total number of occurrences of the part $k$ among all partitions of $n$. For each partition $\lambda$, let $m_{k}(\lambda)$ denote the number of parts in $\lambda$ equal to $k$. Then

$$
\sum_{\lambda \vdash n} \#\{h \in \mathcal{H}(\lambda), h=k\}=\sum_{\lambda \vdash n} k \times m_{k}(\lambda) .
$$

In fact, the following more general result can be deduced from Theorem 7.1. 
Corollary 7.2. For each positive integer $n, t$, and each complex number $\beta$, we have

$$
\sum_{\lambda \vdash n} \sum_{h \in \mathcal{H}_{t}(\lambda)} h^{\beta}=\sum_{\lambda \vdash n} \sum_{k \geq 1}(t k)^{\beta+1} m_{t k}(\lambda) .
$$

We next prove the following theorem that generalizes formula (1.4).

Theorem 7.3. For any positive integer $t$ and complex number $\beta$, we have

$$
\sum_{\lambda \in \mathcal{P}} q^{|\lambda|} \sum_{h \in \mathcal{H}_{t}(\lambda)} h^{\beta}=\prod_{m \geq 1} \frac{1}{1-q^{m}} \times \sum_{k \geq 1}(t k)^{\beta+1} \frac{q^{t k}}{1-q^{t k}} .
$$

By Corollary 7.2, we see that Theorem 7.3 is equivalent to the next theorem.

Theorem 7.4. We have

$$
\sum_{n \geq 1} q^{n} \sum_{\lambda \vdash n} \sum_{k \geq 1}(t k)^{\beta} m_{t k}(\lambda)=\prod_{m \geq 1} \frac{1}{1-q^{m}} \times \sum_{k \geq 1}(t k)^{\beta} \frac{q^{t k}}{1-q^{t k}} .
$$

Proof. Given a fixed positive integer $k$ divisible by $t$, it is known that

$$
\begin{aligned}
\sum_{\lambda \in \mathcal{P}} k^{\beta} m_{k}(\lambda) q^{|\lambda|} & =\left(k^{\beta} q^{k}+2 k^{\beta} q^{2 k}+3 k^{\beta} q^{3 k}+\cdots\right) \prod_{m \neq k} \frac{1}{1-q^{m}} \\
& =\left(k^{\beta-1} q \frac{d}{d q} \frac{q^{k}}{1-q^{k}}\right) \prod_{m \neq k} \frac{1}{1-q^{m}} \\
& =\left(k^{\beta} \frac{q^{k}}{\left(1-q^{k}\right)^{2}}\right) \prod_{m \neq k} \frac{1}{1-q^{m}} \\
& =k^{\beta} \frac{q^{k}}{1-q^{k}} \prod_{m \geq 1} \frac{1}{1-q^{m}} .
\end{aligned}
$$

Hence, we have

$$
\sum_{n \geq 1} q^{n} \sum_{\lambda \vdash n} \sum_{k \geq 1}(t k)^{\beta} m_{t k}(\lambda)=\prod_{m \geq 1} \frac{1}{1-q^{m}} \sum_{k \geq 1}(t k)^{\beta} \frac{q^{t k}}{1-q^{t k}} .
$$

We now give two generalizations of (1.4) by applying Theorems 1.3 and 1.4 with the weight function $\rho(h)=h^{\beta}$. Notice that Theorem 7.5 reduces to Theorem 7.3 when $x=1$. However, it cannot be proved directly using the Bessenrodt-BacherManivel theorem.

Theorem 7.5. For any complex number $\beta$ and positive integer $t$, we have

$$
\sum_{\lambda \in \mathcal{P}} q^{|\lambda|} x^{\# \mathcal{H}_{t}(\lambda)} \sum_{h \in \mathcal{H}_{t}(\lambda)} h^{\beta}=\prod_{k \geq 1} \frac{\left(1-q^{t k}\right)^{t}}{\left(1-\left(x q^{t}\right)^{k}\right)^{t}\left(1-q^{k}\right)} \sum_{k \geq 1}(t k)^{\beta+1} \frac{\left(x q^{t}\right)^{k}}{1-\left(x q^{t}\right)^{k}} .
$$

Theorem 7.6. For any complex number $\beta$, we have

$$
\begin{aligned}
& \sum_{\lambda \in \mathcal{P}} q^{|\lambda|} x^{\# \mathcal{H}_{2}(\lambda)} b^{\mathrm{BG}(\lambda)} \sum_{h \in \mathcal{H}_{2}(\lambda)} h^{\beta} \\
& \quad=\prod_{k \geq 1} \frac{1}{\left(1-\left(x q^{2}\right)^{k}\right)^{2}} \sum_{k \geq 1}(2 k)^{\beta+1} \frac{\left(x q^{2}\right)^{k}}{1-\left(x q^{2}\right)^{k}} \times \sum_{j=-\infty}^{+\infty} b^{j} q^{j(2 j-1)} .
\end{aligned}
$$


The specializations $\beta=1$ and $\beta=-1$ of Theorems 7.5 and 7.6 are worth mentioning and are not reproduced. A further specialization with $\beta=-1, t=$ $2, x=1 / q$ implies Corollary 1.11 .

\section{The Okada-Panova formula}

Formula (1.7) is the generating function form of the Okada-Panova formula (8.1), which was conjectured by Okada and proved by Panova [21, 23]:

$$
\frac{1}{n !} \sum_{\lambda \vdash n} f_{\lambda}^{2} \sum_{h \in \mathcal{H}(\lambda)} \prod_{i=1}^{r}\left(h^{2}-i^{2}\right)=C(r) \prod_{j=0}^{r}(n-j),
$$

where $C(r)$ is defined in (1.7). By the hook length formula,

$$
f_{\lambda}=\frac{n !}{\prod_{h \in \mathcal{H}(\lambda)} h},
$$

formula (8.1) can be written as

$$
\sum_{\lambda \vdash n} \prod_{h \in \mathcal{H}(\lambda)} \frac{1}{h^{2}} \sum_{h \in \mathcal{H}(\lambda)} \prod_{i=1}^{r}\left(h^{2}-i^{2}\right)=C(r) \frac{1}{(n-r-1) !} .
$$

It is easy to see that formula (1.7) is the generating function version of the above formula.

We now give a generalization of the Okada-Panova formula (1.7) by using Theorems 1.1 and 1.2 with the weight functions $\rho_{1}(h)=\frac{1}{h^{2}}$ and $\rho_{2}(h)=\prod_{i=1}^{r}\left(h^{2}-i^{2}\right)$. By the classical hook formula (1.1), it is known that

$$
f_{\alpha}(q)=\sum_{\lambda \in \mathcal{P}} q^{|\lambda|} \prod_{h \in \mathcal{H}(\lambda)} \frac{1}{(\alpha h)^{2}}=\exp \left(\frac{q}{\alpha^{2}}\right) .
$$

Recall that

$$
g_{\alpha}(q)=\sum_{\lambda \in \mathcal{P}} q^{|\lambda|} \prod_{h \in \mathcal{H}(\lambda)} \frac{1}{(\alpha h)^{2}} \sum_{h \in \mathcal{H}(\lambda)} \prod_{i=1}^{r}\left((\alpha h)^{2}-i^{2}\right) .
$$

To evaluate $g_{\alpha}(q)$ we introduce a family of polynomials $\left(B_{r, k}(\alpha)\right)_{0 \leq k \leq r}$ defined by the following relations:

$$
\begin{aligned}
& B_{r, 0}(\alpha)=\prod_{j=1}^{r}\left(\alpha^{2}-j^{2}\right), \\
& B_{r, k}(\alpha)=\left[\alpha^{2}(k+1)^{2}-r^{2}\right] B_{r-1, k}(\alpha)+\alpha^{2} B_{r-1, k-1}(\alpha) \\
& \quad \text { for } 1 \leq k \leq r-1, \\
& B_{r, r}(\alpha)=\alpha^{2 r} .
\end{aligned}
$$

The first values of the polynomials $B_{r, k}(\alpha)$ are:

$$
\begin{aligned}
& B_{1,0}(\alpha)=\alpha^{2}-1, B_{1,1}(\alpha)=\alpha^{2}, \\
& B_{2,0}(\alpha)=\left(\alpha^{2}-1\right)\left(\alpha^{2}-2^{2}\right), B_{2,1}(\alpha)=5 \alpha^{2}\left(\alpha^{2}-1\right), B_{2,2}(\alpha)=\alpha^{4}, \\
& B_{3,0}(\alpha)=\left(\alpha^{2}-1\right)\left(\alpha^{2}-2^{2}\right)\left(\alpha^{2}-3^{2}\right), B_{3,1}(\alpha)=7 \alpha^{2}\left(\alpha^{2}-1\right)\left(3 \alpha^{2}-7\right), \\
& B_{3,2}(\alpha)=14 \alpha^{4}\left(\alpha^{2}-1\right), B_{3,3}(\alpha)=\alpha^{6} .
\end{aligned}
$$


Lemma 8.1. For any complex number $\alpha$, we have

$$
\prod_{j=1}^{r}\left(\alpha^{2} x^{2}-j^{2}\right)=\sum_{k=0}^{r} B_{r, k}(\alpha) \prod_{j=1}^{k}\left(x^{2}-j^{2}\right) .
$$

Proof. Let $p_{r}(\alpha)=\prod_{j=1}^{r}\left(\alpha^{2} x^{2}-j^{2}\right)$ and write

$$
p_{r}(\alpha)=\sum_{k=0}^{r} B_{r, k}^{\prime}(\alpha) p_{k}(1)
$$

We need to show that $\left(B_{r, k}^{\prime}(\alpha)\right)$ satisfy the same relations as $\left(B_{r, k}(\alpha)\right)$. From the definition of $p_{r}(\alpha)$, we have

$$
\begin{aligned}
p_{r}(\alpha) & =\left(\alpha^{2} x^{2}-r^{2}\right) \sum_{k=0}^{r-1} B_{r-1, k}^{\prime}(\alpha) p_{k}(1) \\
& =\sum_{k=0}^{r-1} B_{r-1, k}^{\prime}(\alpha)\left[\alpha^{2}\left(x^{2}-(k+1)^{2}\right)+\alpha^{2}(k+1)^{2}-r^{2}\right] p_{k}(1) \\
& =\alpha^{2} \sum_{k=0}^{r-1} B_{r-1, k}^{\prime}(\alpha) p_{k+1}(1)+\sum_{k=0}^{r-1}\left[\alpha^{2}(k+1)^{2}-r^{2}\right] B_{r-1, k}^{\prime}(\alpha) p_{k}(1) \\
& =\alpha^{2} \sum_{k=1}^{r} B_{r-1, k-1}^{\prime}(\alpha) p_{k}(1)+\sum_{k=0}^{r-1}\left[\alpha^{2}(k+1)^{2}-r^{2}\right] B_{r-1, k}^{\prime}(\alpha) p_{k}(1) .
\end{aligned}
$$

Since the $p_{k}(1)$ 's are linearly independent, we obtain the following recurrences:

$$
B_{r, r}^{\prime}(\alpha)=\alpha^{2} B_{r-1, r-1}^{\prime}(\alpha), B_{r, 0}^{\prime}(\alpha)=\left(\alpha^{2}-r^{2}\right) B_{r-1,0}^{\prime}(\alpha)
$$

and for $0<k<r$,

$$
B_{r, k}^{\prime}(\alpha)=\alpha^{2} B_{r-1, k-1}^{\prime}(\alpha)+\left[\alpha^{2}(k+1)^{2}-r^{2}\right] B_{r-1, k}^{\prime}(\alpha) .
$$

Iterating recurrence (8.4) $(r-1)$ times and using the fact that $B_{0,0}^{\prime}(\alpha)=1$, we obtain $B_{r, r}^{\prime}(\alpha)=\alpha^{2 r}, B_{r, 0}^{\prime}(\alpha)=\prod_{j=1}^{r}\left(\alpha^{2}-j^{2}\right)$.

Thus we can express the series $g_{\alpha}(q)$ from Lemma 8.1 and Okada-Panova (1.7) as follows.

Proposition 8.2. We have

$$
\begin{aligned}
g_{\alpha}(q) & =\sum_{\lambda \in \mathcal{P}} q^{|\lambda|} \prod_{h \in \mathcal{H}(\lambda)} \frac{1}{(\alpha h)^{2}} \sum_{h \in \mathcal{H}(\lambda)} \prod_{i=1}^{r}\left((\alpha h)^{2}-i^{2}\right) \\
& =\exp \left(\frac{q}{\alpha^{2}}\right) \sum_{k=0}^{r} B_{r, k}(\alpha) C(k)\left(\frac{q}{\alpha^{2}}\right)^{k+1} .
\end{aligned}
$$


Proof.

$$
\begin{aligned}
g_{\alpha}(q) & =\sum_{\lambda \in \mathcal{P}}\left(\frac{q}{\alpha^{2}}\right)^{|\lambda|} \prod_{h \in \mathcal{H}(\lambda)} \frac{1}{h^{2}} \sum_{h \in \mathcal{H}(\lambda)} \prod_{i=1}^{r}\left((\alpha h)^{2}-i^{2}\right) \\
& =\sum_{\lambda \in \mathcal{P}}\left(\frac{q}{\alpha^{2}}\right)^{|\lambda|} \prod_{h \in \mathcal{H}(\lambda)} \frac{1}{h^{2}} \sum_{h \in \mathcal{H}(\lambda)} \sum_{k=0}^{r} B_{r, k}(\alpha) \prod_{j=1}^{k}\left(h^{2}-j^{2}\right) \\
& =\sum_{k=0}^{r} B_{r, k}(\alpha) \sum_{\lambda \in \mathcal{P}}\left(\frac{q}{\alpha^{2}}\right)^{|\lambda|} \prod_{h \in \mathcal{H}(\lambda)} \frac{1}{h^{2}} \sum_{h \in \mathcal{H}(\lambda)} \prod_{j=1}^{k}\left(h^{2}-j^{2}\right) \\
& =\sum_{k=0}^{r} B_{r, k}(\alpha) C(k)\left(\frac{q}{\alpha^{2}}\right)^{k+1} \exp \left(\frac{q}{\alpha^{2}}\right)
\end{aligned}
$$

where the last equation follows from (1.7).

From the definition of $B_{r, k}(\alpha)$ in Lemma 8.1, it is known that for any positive integer $t, B_{r, k}(t)=0$ for $r \geq t(k+1)$. Combining (8.3) and (8.5), we see that Theorems 1.1 and 1.2 give the following generalizations of Okada-Panova's formula (1.7).

Theorem 8.3. For any positive integer $t$ and $r$, we have

$$
\begin{aligned}
& \sum_{\lambda \in \mathcal{P}} q^{|\lambda|} x^{\# \mathcal{H}_{t}(\lambda)} \prod_{h \in \mathcal{H}_{t}(\lambda)} \frac{1}{h^{2}} \sum_{h \in \mathcal{H}_{t}(\lambda)} \prod_{i=1}^{r}\left(h^{2}-i^{2}\right) \\
& \quad=t \exp \left(\frac{x q^{t}}{t}\right) \prod_{k \geq 1} \frac{\left(1-q^{t k}\right)^{t}}{1-q^{k}} \sum_{k=\left\lceil\frac{r-t+1}{t}\right\rceil}^{r} B_{r, k}(t) C(k)\left(\frac{x q^{t}}{t^{2}}\right)^{k+1} .
\end{aligned}
$$

Theorem 8.4. For any positive integer $r$, we have

$$
\begin{aligned}
& \sum_{\lambda \in \mathcal{P}} q^{|\lambda|} x^{\# \mathcal{H}_{2}(\lambda)} b^{\mathrm{BG}(\lambda)} \prod_{h \in \mathcal{H}_{2}(\lambda)} \frac{1}{h^{2}} \sum_{h \in \mathcal{H}_{2}(\lambda)} \prod_{i=1}^{r}\left(h^{2}-i^{2}\right) \\
& \quad=2 \exp \left(\frac{x q^{2}}{2}\right) \sum_{j=-\infty}^{+\infty} b^{j} q^{j(2 j-1)} \sum_{k=\left\lceil\frac{r-1}{2}\right\rceil}^{r} B_{r, k}(2) C(k)\left(\frac{x q^{2}}{4}\right)^{k+1} .
\end{aligned}
$$

\section{The Stanley-Panova formula}

In [13, the first author conjectured that

$$
\frac{1}{n !} \sum_{\lambda \vdash n} f_{\lambda}^{2} \sum_{h \in \mathcal{H}(\lambda)} h^{2 k}
$$

is a polynomial function in $n$ of degree $k+1$. Stanley and Panova proved a generalization of this conjecture and deduced the following explicit formula of this summation from Okada's conjecture [21, 23]:

$$
\frac{1}{n !} \sum_{\lambda \vdash n} f_{\lambda}^{2} \sum_{h \in \mathcal{H}(\lambda)} h^{2 k}=\sum_{i=0}^{k} T(k+1, i+1) C(i) \prod_{j=0}^{i}(n-j),
$$


where $T(k, i)$ is a central factorial number [22, ex.5.8] defined for $k \geq 1$ and $i \geq 1$ by

$$
\begin{aligned}
& T(k, 0)=T(0, i)=0, \quad T(1,1)=1, \\
& T(k, i)=i^{2} T(k-1, i)+T(k-1, i-1) \text { for }(k, i) \neq(1,1) .
\end{aligned}
$$

By the hook length formula (8.2) one can easily see that formula (1.8) is the generating function form of formula (9.1).

We now give two generalizations of Stanley-Panova's formula (1.8) by using Theorems 1.1 and 1.2 with the weight functions $\rho_{1}(h)=1 / h^{2}$ and $\rho_{2}(h)=h^{2 k}$. By the classical hook formula (1.1) we know that

$$
f_{\alpha}(q)=\sum_{\lambda \in \mathcal{P}} q^{|\lambda|} \prod_{h \in \mathcal{H}(\lambda)} \frac{1}{(\alpha h)^{2}}=\exp \left(\frac{q}{\alpha^{2}}\right) .
$$

From the Stanley-Panova formula (1.8) it is known that

$$
\begin{aligned}
g_{\alpha}(q) & =\sum_{\lambda \in \mathcal{P}} q^{|\lambda|} \prod_{h \in \mathcal{H}(\lambda)} \frac{1}{(\alpha h)^{2}} \sum_{h \in \mathcal{H}(\lambda)} \alpha^{2 k} h^{2 k} \\
& =\alpha^{2 k} \exp \left(\frac{q}{\alpha^{2}}\right) \sum_{i=0}^{k} T(k+1, i+1) C(i)\left(\frac{q}{\alpha^{2}}\right)^{i+1} .
\end{aligned}
$$

Thus, Theorems 1.1 and 1.2 imply the following generalizations of Stanley-Panova's formula (1.8).

Theorem 9.1. For any positive integers $t$ and $k$, we have

$$
\begin{aligned}
& \sum_{\lambda \in \mathcal{P}} q^{|\lambda|} x^{\# \mathcal{H}_{t}(\lambda)} \prod_{h \in \mathcal{H}_{t}(\lambda)} \frac{1}{h^{2}} \sum_{h \in \mathcal{H}_{t}(\lambda)} h^{2 k} \\
& =t^{2 k+1} \exp \left(\frac{x q^{t}}{t}\right) \prod_{j \geq 1} \frac{\left(1-q^{t j}\right)^{t}}{1-q^{j}} \sum_{i=0}^{k} T(k+1, i+1) C(i)\left(\frac{x q^{t}}{t^{2}}\right)^{i+1} .
\end{aligned}
$$

Theorem 9.2. For any positive integer $k$, we have

$$
\begin{aligned}
& \sum_{\lambda \in \mathcal{P}} q^{|\lambda|} x^{\# \mathcal{H}_{2}(\lambda)} b^{\mathrm{BG}(\lambda)} \prod_{h \in \mathcal{H}_{2}(\lambda)} \frac{1}{h^{2}} \sum_{h \in \mathcal{H}_{2}(\lambda)} h^{2 k} \\
& =2^{2 k+1} \exp \left(\frac{x q^{2}}{2}\right) \sum_{j=-\infty}^{+\infty} b^{j} q^{j(2 j-1)} \sum_{i=0}^{k} T(k+1, i+1) C(i)\left(\frac{x q^{2}}{4}\right)^{i+1} .
\end{aligned}
$$

\section{REFERENCES}

1. Ron M. Adin and Avital Frumkin, Rim hook tableaux and Kostant's $\eta$-function coefficients, Adv. Appl. Math. 33 (2004) 492-511. MR2081040 (2005f:05165)

2. George E. Andrews, The Theory of Partitions, Addison-Wesley, Reading, 1976. MR0557013 $(58: 27738)$

3. Christine Bessenrodt, On hooks of Young diagrams, Ann. Comb. 2 (1998) 103-110. MR.1682922 (2000d:05126)

4. Alexander Berkovich and Frank G. Garvan, On the Andrews-Stanley refinement of Ramanujan's partition congruence modulo 5 and generalizations, Trans. Amer. Math. Soc. 358 (2006) 703-726. MR2177037 (2006g:11212)

5. Roland Bacher and Laurent Manivel, Hooks and powers of parts in partitions, Sém. Lothar. Combin. 47 (2001) article B47d. MR1894024 (2003c:05020) 
6. Kevin Carde, Joe Loubert, Aaron Potechin, and Adrian Sanborn, Proof of Han's hook expansion conjecture, arXiv:0808.0928, 2008.

7. William Y.C. Chen, Kathy Q. Ji, and Herbert S. Wilf, BG-ranks and 2-cores, Electron. J. Combin. 13 (2006) N18. MR2274300 (2009a:05010)

8. G. Gaspar and M. Rahman, Basic Hypergeometric Series, Cambridge University Press, 1990. MR.1052153 (91d:33034)

9. Frank Garvan, Dongsu Kim, and Dennis Stanton, Cranks and t-cores, Invent. Math. 101 (1990) 1-17. MR.1055707 (91h:11106)

10. Guo-Niu Han, An explicit expansion formula for the powers of the Euler Product in terms of partition hook lengths, arXiv:0804.1849v2, 2008.

11. Guo-Niu Han, Some conjectures and open problems about partition hook length, Experimental Mathematics 18 (2009) 97-106. MR2548990

12. Guo-Niu Han, The Nekrasov-Okounkov hook length formula: Refinement, elementary proof, extension and applications, Annales de I'Institut Fourier 60 (2010) 1-29. MR2664308

13. Guo-Niu Han, Discovering hook length formulas by an expansion technique, Electron. J. Combin. 15 (2008) R133. MR2448883

14. Gordon James and Adalbert Kerber, The Representation Theory of the Symmetric Group, Encyclopedia of Mathematics and its Applications 16, Addison-Wesley Publishing, Reading, MA, 1981. MR644144 (83k:20003)

15. Donald E. Knuth, The Art of Computer Programming, vol.3, Sorting and Searching, 2nd ed., Addison Wesley Longman, 1998. MR0445948 (56:4281)

16. Alain Lascoux, Symmetric Functions and Combinatorial Operators on Polynomials, CBMS Regional Conference Series in Mathematics, 99, 2001. MR2017492 (2005b:05217)

17. D. E. Littlewood, Modular representations of symmetric groups, Proc. Roy. Soc. London. Ser. A 209 (1951) 333-353. MR0049896 (14:243b)

18. Ian G. Macdonald, Symmetric Functions and Hall Polynomials, Second Edition, Clarendon Press, Oxford, 1995. MR1354144 (96h:05207)

19. T. Nakayama, On some modular properties of irreducible representations of a symmetric group, I. II, Japan. J. Math. 17 (1941) 89-108, 411-423. MR0005729 (3:195d) MR0005730 $(3: 196 \mathrm{a})$

20. Nikita A. Nekrasov and Andrei Okounkov, Seiberg-Witten theory and random partitions, The unity of mathematics, 525-596, Progr. Math. 244, Birkhäuser Boston, 2006. MR2181816 (2008a:81227)

21. Greta Panova, Proof of a conjecture of Okada, arXiv:0811.3463, 2008.

22. Richard P. Stanley, Enumerative Combinatorics, vol. 2, Cambridge University Press, 1999. MR $1676282(2000 \mathrm{k}: 05026)$

23. Richard P. Stanley, Some combinatorial properties of hook lengths, contents, and parts of partitions, Ramanujan J., to appear, 2009.

I.R.M.A., UMR 7501, Université de Strasbourg et CNRS, 7 rue René-Descartes, F67084 Strasbourg, France

E-mail address: guoniu@math.u-strasbg.fr

Center for Combinatorics, LPMC-TJKLC, Nankai University, Tianjin 300071, PeoPLE'S REPUBLIC OF ChINA

E-mail address: ji@nankai.edu.cn 